\title{
Potent Activities of Roemerine against Candida albicans and the Underlying Mechanisms
}

\section{Chaoyu Ma ${ }^{1,2, \dagger}$, Faya Du ${ }^{1, \dagger}$, Lan Yan ${ }^{3, \dagger}$, Gonghao He ${ }^{1}$, Jianchang He ${ }^{1}$, Chengying Wang ${ }^{4}$, Gaoxiong Rao ${ }^{4}$, Yuanying Jiang ${ }^{3, *}$ and Guili $\mathrm{Xu}{ }^{1, *}$}

1 Department of Pharmacy, Kunming General Hospital of Chengdu Military Region, 212 Da-Guan Road, Kunming 650032, China; E-Mails: 15198808817@163.com (C.M.); faya_du@126.com (F.D.); hegonghao@163.com (G.H.); hejianchang123@sina.com (J.H.)

2 School of Pharmacy, Kunming Medical University, 1168 Yu-Hua Road, Kunming 650500, China

3 New Drug Research and Development Center, School of Pharmacy, Second Military Medical University, Shanghai 200433, China; E-Mail: y108101963@163.com

4 School of Pharmacy, Yunnan College of Traditional Chinese Medicine, 1076 Yu-Hua Road, Kunming 650500, China; E-Mails: wcy19901111@126.com (C.W.); raogaoxiong@yeah.net (G.R.)

$\dagger$ These authors contributed equally to this work.

* Authors to whom correspondence should be addressed; E-Mails: 13761571578@163.com (Y.J.); xug1_0044@163.com (G.X.); Tel.: +86-021-8187-1357 (Y.J.); Fax: +86-021-8187-1275 (Y.J.); Tel./Fax: +86-871-6477-4784 (G.X.).

Academic Editor: Derek J. McPhee

Received: 1 August 2015 / Accepted: 22 September 2015 / Published: 29 September 2015

\begin{abstract}
Roemerine (RM) is an aporphine alkaloid isolated from the fresh rattan stem of Fibraurea recisa, and it has been demonstrated to have certain antifungal activity. This study aimed to investigate the antifungal activity of RM and the underlying mechanisms in Candida albicans (C. albicans). The in vitro antifungal activity of RM was evaluated by a series of experiments, including the XTT reduction assay, confocal laser scanning microscopy assay, scanning electron microscope assay. Results showed that $1 \mu \mathrm{g} / \mathrm{mL}$ RM inhibited biofilm formation significantly $(p<0.01)$ both in Spider medium and Lee's medium. In addition, RM could inhibit yeast-to-hyphae transition of $C$. albicans in a dose-dependent manner. The biofilm-specific and hypha-specific genes such as YWP1, SAP5, SAP6, HWP1, ECE1 were up-regulated and $E F G 1$ was down-regulated after $8 \mu \mathrm{g} / \mathrm{mL}$ RM treatment. Furthermore, the toxicity of RM was investigated using $C$. elegans worms, three cancer cells and one normal cell. The date showed that RM had no significant toxicity. In conclusion, RM could inhibited
\end{abstract}


the formation of C. albicans biofilm in vitro, but it had no fungicidal effect on planktonic C. albicans cells, and the anti-biofilm mechanism may be related to the cAMP pathway.

Keywords: antifungal activity; roemerine; Candida albicans; biofilm

\section{Introduction}

Candida albicans (C. albicans) is the most common fungal pathogen, which has a high propensity to cause superficial or life-threatening invasive infections in immunocompromised patients [1]. Although the need for effective antifungal therapy is increasing, the number of available antifungal agents is still limited. In addition, with the increasing clinical use of these limited antifungal agents, drug-resistant isolates are emerging rapidly [2].

A striking feature of $C$. albicans is its ability to grow in three morphological forms: budding yeast, pseudohyphal and hyphal forms [3]. Besides, C. albicans easily develops into a biofilm on the surfaces of almost any medical device, resulting in biofilm-associated infections [4]. Biofilms are structured microbial communities that are attached to a surface and are embedded in a matrix of exopolymeric material [5]. Comparing with planktonic cells, $C$. albicans biofilms display more severe resistance to a wide variety of clinical antifungal agents [6]. Therefore, new antifungal agents and strategies are urgently needed to address these challenges.

Roemerine ( $\mathrm{RM})$ is an aporphine alkaloid (Figure 1). It is isolated from the fresh rattan stem of Fibraurea recisa. This bioactive alkaloid has a variety of pharmacological properties, such as vasodilator [7], anthelmintic [8] and antiplasmodial activities [9]. It also enhances the cytotoxicity of vinblastine against multidrug-resistant KB-V1 cells [10] and has high affinity for the 5-HT2A receptor [11]. In addition, it was reported that (+)-roemerine MeI, one of the derivatives of RM, had strong antibacterial activity against G+ bacteria, including Bacillus cereus, Micrococcus sp. and Staphylococcus aureus [12]. Besides, our previous studies showed that RM had a certain activity against fungal pathogens, such as Candida albicans, Candida glabrata, Candida krusei, Candida parapsilosis, and Cryptococcus neoformans [13]. Moreover, we also found that RM had great bioavailability (84\%) in Sprague-Dawley (SD) rats, and it was suggested that RM could be taken orally instead of intravenously [14]. Based on this background, RM may be a promising drug against fungal pathogens. Development of RM as a new antifungal agent would be a significant advance in clinical treatment of fungus infection. Nevertheless, the effect of RM against $C$. albicans biofilms and the underlying mechanisms have not yet been investigated.

In recent years, several signal transduction pathways like cyclic AMP (cAMP) pathway and mitogen-activated protein kinase (MAPK) pathway and some key transcription factors have been intensively investigated to uncover the mechanisms of C. albicans morphological transition. In these signal transduction pathways, some adhesion-, hypha-, and biofilm-specific genes, including ECE1, RAS1, HWP1, YWP1, UME6, EAP1, ALS3, BCY1, EFG1, CSH1, CYR1, HGC1, CPH1 and PDE2 play essential roles. Efg1 and $\mathrm{Cph} 1$ are transcription factors of cAMP and MAPK signaling pathways, respectively. However, Ras1 stimulates both the cAMP and MAPK pathways $[15,16]$. In this study, we evaluated the activities of RM against $C$. albicans biofilms in vitro, and exploring the underlying mechanisms, and we found that the antibiofilm mechanism is associated with the cAMP pathway. 


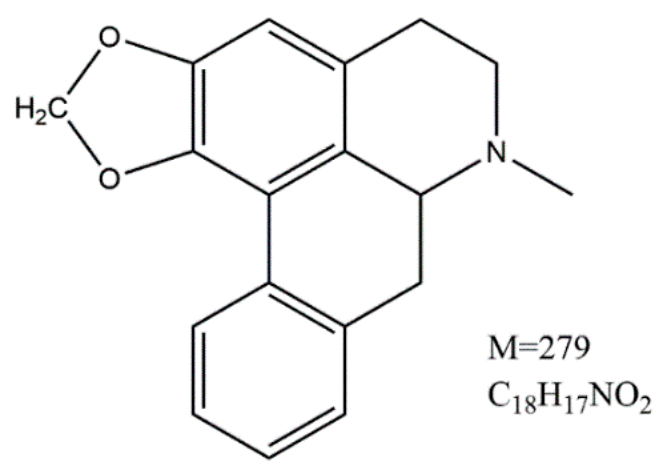

Figure 1. Chemical structure of RM, which is an aporphine alkaloid isolated from the fresh rattan stem of Fibraurea recisa.

\section{Results}

\subsection{Effect of RM against C. albicans Yeast Cells}

The in vitro antimicrobial activity of RM was evaluated by broth microdilution assay, and the antimicrobial spectrum is demonstrated in Table 1. RM alone exhibited weak effect against $C$. albicans (MIC80: 128-256 mg/L), while it showed more potent activity against Candida glabrata, Candida krusei, Candida tropicalis, Candida parapsilosis, Aspergillus fumigatus and Staphylococcus aureus (Table 1). Meanwhile, the effect of different concentrations of RM on growth of C. albicans SC5314 was further established by time-growth curves (Figure 2). It showed that at concentrations ranging from 2 to $128 \mu \mathrm{g} / \mathrm{mL}$, RM could not significantly inhibit the growth of $C$. albicans, the yeast cells growth was similar to that of the control group without RM treatment. By contrast, $256 \mu \mathrm{g} / \mathrm{mL}$ RM showed a significant effect on C. albicans growth (Figure 2).

Table 1. The antimicrobial spectrum of RM (MIC 50 s and $\mathrm{MIC}_{80 \mathrm{~s}}$ in $\left.\mathrm{mg} / \mathrm{L}\right)$.

\begin{tabular}{|c|c|c|}
\hline Strains & $\mathrm{RM}\left(\mathrm{MIC}_{50 \mathrm{~s}}, \mathrm{mg} / \mathrm{L}\right)$ & $\mathrm{RM}\left(\mathrm{MIC}_{80 \mathrm{~s}}, \mathrm{mg} / \mathrm{L}\right)$ \\
\hline Candida albicans SC5314 & 128 & 256 \\
\hline Candida glabrata 8535 & 32 & 64 \\
\hline Candida krusei 4996 & 16 & 32 \\
\hline Candida tropicalis 8915 & 32 & 64 \\
\hline Candida parapsilosis 90018 & 16 & 32 \\
\hline Aspergillus fumigatus 7544 & 16 & 32 \\
\hline$*$ MRSA 135 & 32 & 64 \\
\hline MRSA 98 & 32 & 64 \\
\hline MRSA 310 & 16 & 32 \\
\hline MRSA 311 & 32 & 64 \\
\hline MRSA 111 & 32 & 64 \\
\hline MRSA 234 & 32 & 64 \\
\hline MRSA 92 & 32 & 64 \\
\hline MRSA 8 & 32 & 64 \\
\hline Staphylococcus aureus ATCC 25913 & 32 & 64 \\
\hline
\end{tabular}

* Methicillin-resistant Staphylococcus aureus, MRSA. 


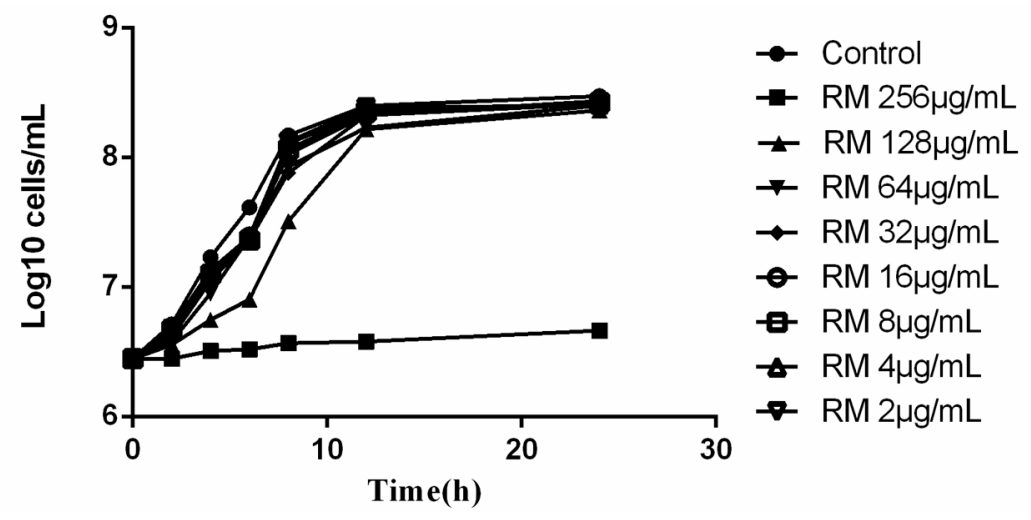

Figure 2. Effects of different concentrations of RM on fungal growth of C. albican SC5314. Exponentially growing C. albicans were suspended in YPD medium to the starting OD 600 of 0.2 (about $2.5 \times 10^{6}$ cells $/ \mathrm{mL}$ ). Then, the suspensions were added in different concentrations of RM and cultured at $30^{\circ} \mathrm{C}, 200 \mathrm{rpm}$. C. albicans suspended in YPD medium with $1 \% \mathrm{DMSO}$ was used as the control. Cells were counted at the predetermined time points $(0,2,4,6,8$, $12,24 \mathrm{~h})$.

\subsection{RM Inhibits the Formation of C. albicans Biofilms}

To validate that RM has the anti-biofilm effect, we evaluated XTT reduction assay. In this study, we found that RM inhibited C. albicans biofilm formation in a dose-dependent manner (Figure 3A). In addition, one microgram per milliliter of RM inhibited biofilm formation significantly $(p<0.01)$ both in Spider medium and Lee's medium. The anti-biofilm activity of $8 \mu \mathrm{g} / \mathrm{mL} \mathrm{RM}$ in the Spider medium was the strongest and the $C$. albicans SC5314 biofilm formation was inhibited by $90 \%$ compared with the control. RM inhibited biofilm formation by $80 \%$ in the concentration in $8 \mu \mathrm{g} / \mathrm{mL}$ in Lee's medium (Figure 3A). Furthermore, the anti-biofilm effect of RM was evaluated by CLSM (Figure 3B) and SEM (Figure 3C). C. albicans biofilm formation was disrupted by RM in a dose-dependent manner. In the drug free medium, $C$. albicans cells formed biofilm with a dense network of yeasts and true hyphae (Figure 3(Ba-c,Ca-c)). When cells exposed to $8 \mu \mathrm{g} / \mathrm{mL} \mathrm{RM}$, the biofilm formation was seriously disrupted (Figure 3(Bg-i,Cg-i)). Moreover, cell density was further reduced by $16 \mu \mathrm{g} / \mathrm{mL}$ RM (Figure 3(Bj-1,Cj-1)).

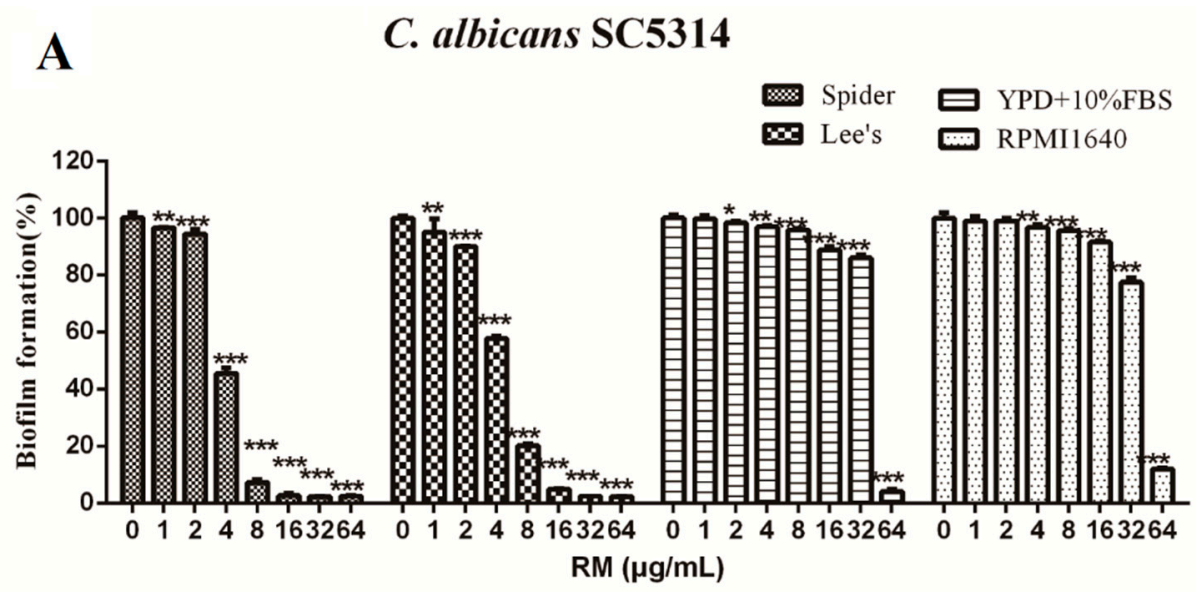

Figure 3. Cont. 


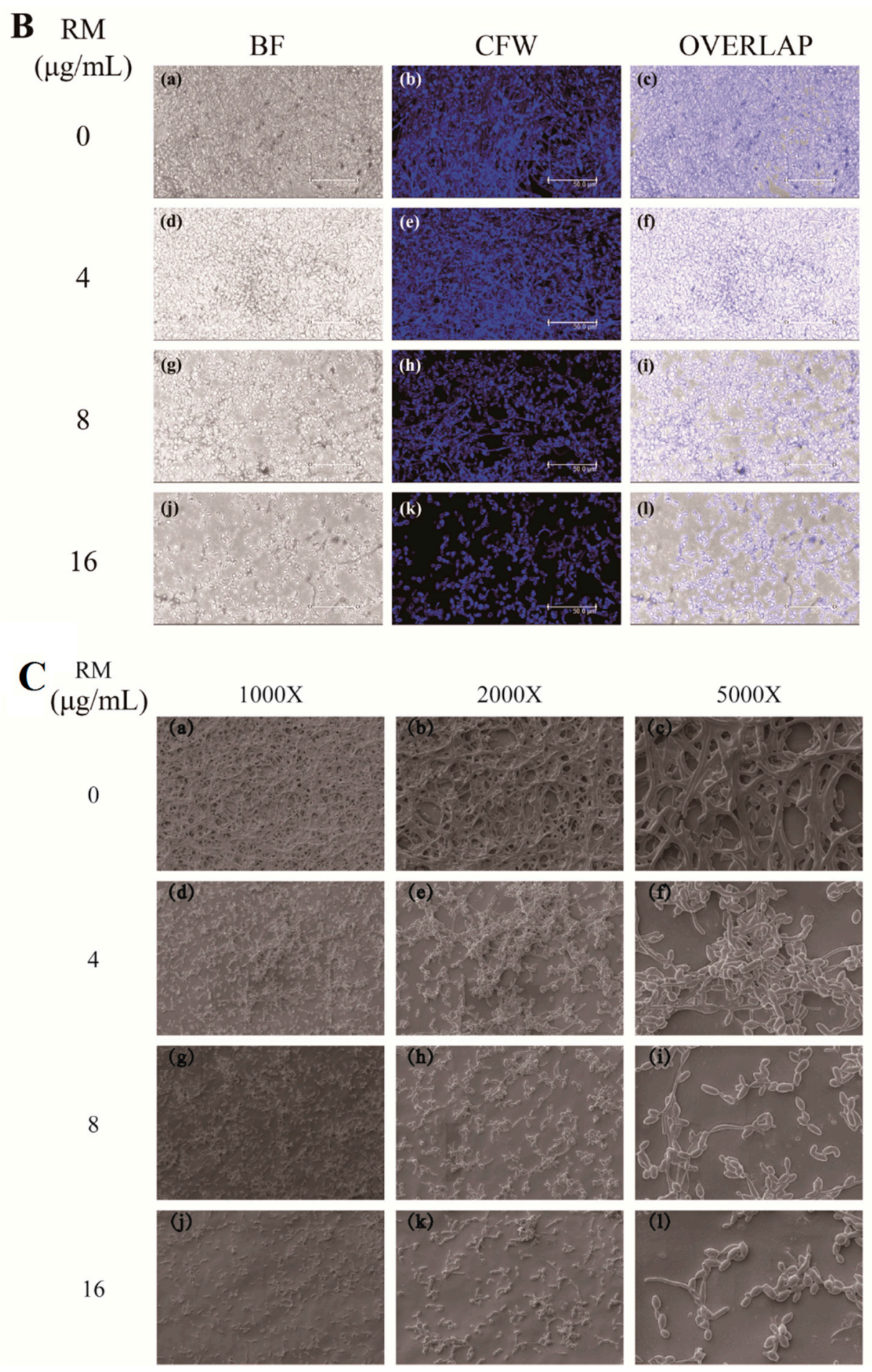

Figure 3. RM inhibits $C$. albicans SC5314 biofilm formation in vitro. (A) Inhibitory activity of different concentrations of RM on biofilm formation in four mediums (Spider, Lee's, RPMI1640 and YPD + 10\% FBS). * $p<0.05 ; * * p<0.01 ; * * * p<0.001$, compared to the control biofilms; (B) Effects of different concentrations of PTE on biofilm formation in Lee's medium, shown in CLSM images. CFW, calcofluor white. Bar $=25 \mu \mathrm{m} ;(\mathbf{C})$ Effects of different concentrations of PTE on biofilm formation in Spider medium, shown in SEM images. The inset in the $1000 \times, 2000 \times$ and $5000 \times$ panels show the area that was magnificated. $(\mathbf{a}-\mathbf{c})$ : normal biofilm; (d-f): cells were treated with $4 \mu \mathrm{g} / \mathrm{mL} \mathrm{RM;} \mathrm{(g-i):} 8 \mu \mathrm{g} / \mathrm{mL} \mathrm{RM}$; (j-l): $16 \mu \mathrm{g} / \mathrm{mL} \mathrm{RM}$. 


\subsection{RM Inhibits the Yeast-to-Hypha Morphological Transition of C. albicans}

To evaluate the effect of RM on the yeast-to-hypha morphological transition of C. albicans, three hypha-inducing mediums (liquid and solid media) - Spider, Lee's, and YPD + 10\% FBS - were used. RM showed strong hypha inhibition activity in different liquid medium (Figure 4A-C, Figure S1), the strong-to-weak sequence of the effect of RM against $C$. albicans biofilms was Spider medium, Lee's medium and YPD $+10 \%$ FBS medium. In liquid Spider medium, one microgram per milliliter of RM could inhibit the growth of hypha, but the effect declined after $6 \mathrm{~h}$, and this effect of RM on yeast-to-hypha transition enhanced with increasing RM concentrations. Four micrograms per milliliter of RM could inhibit the growth of hypha to $48 \mathrm{~h}$, and $32 \mu \mathrm{g} / \mathrm{mL}$ RM could completely inhibit the hyphal growth (Figure 4A). In liquid Lee's medium, one microgram per milliliter of RM could inhibit the growth of hypha, and the effect could continue after $48 \mathrm{~h}$. Likewise, $32 \mu \mathrm{g} / \mathrm{mL}$ RM could completely inhibit the hyphal growth (Figure 4B). The $C$. albicans hyphal growth in liquid serum-containing medium was inhibited by $32 \mu \mathrm{g} / \mathrm{mL}$ RM (Figure 4C). Similarly, only smooth-edged colonies were observed on Spider $(8 \mu \mathrm{g} / \mathrm{mL})$ and Lee's $(2 \mu \mathrm{g} / \mathrm{mL})$ solid medium (Figure 4D). When growing on serum-containing solid medium, colonies appeared smooth and round at $8 \mu \mathrm{g} / \mathrm{mL}$, whereas, the colonies on the control plate were highly wrinkled and uneven (Figure S5). The results supported our hypothesis that RM could inhibit hyphal growth.

A

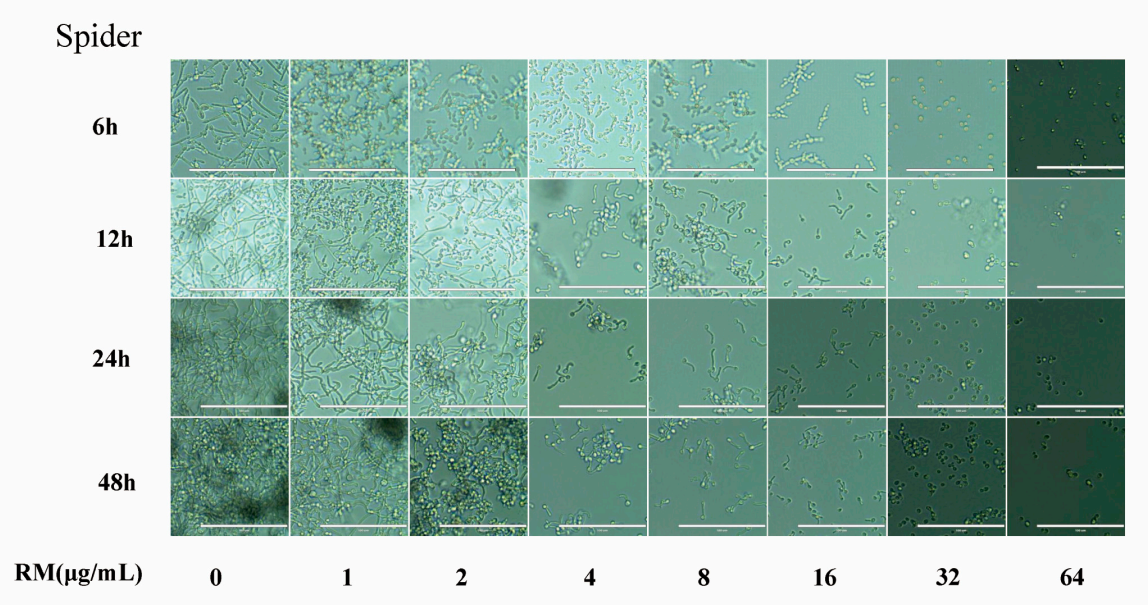

B

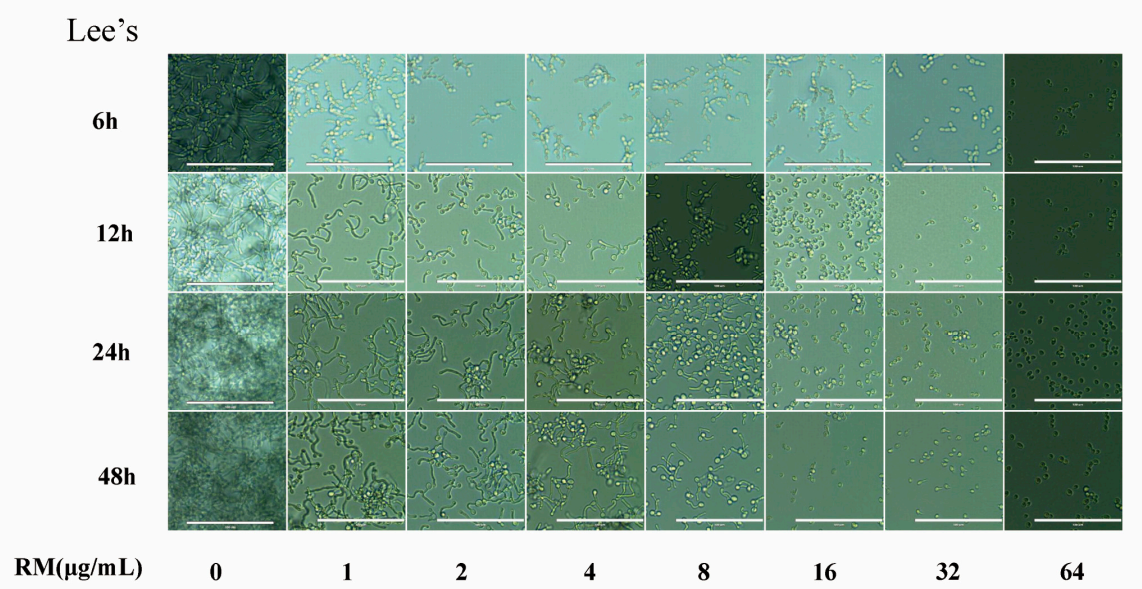

Figure 4. Cont. 


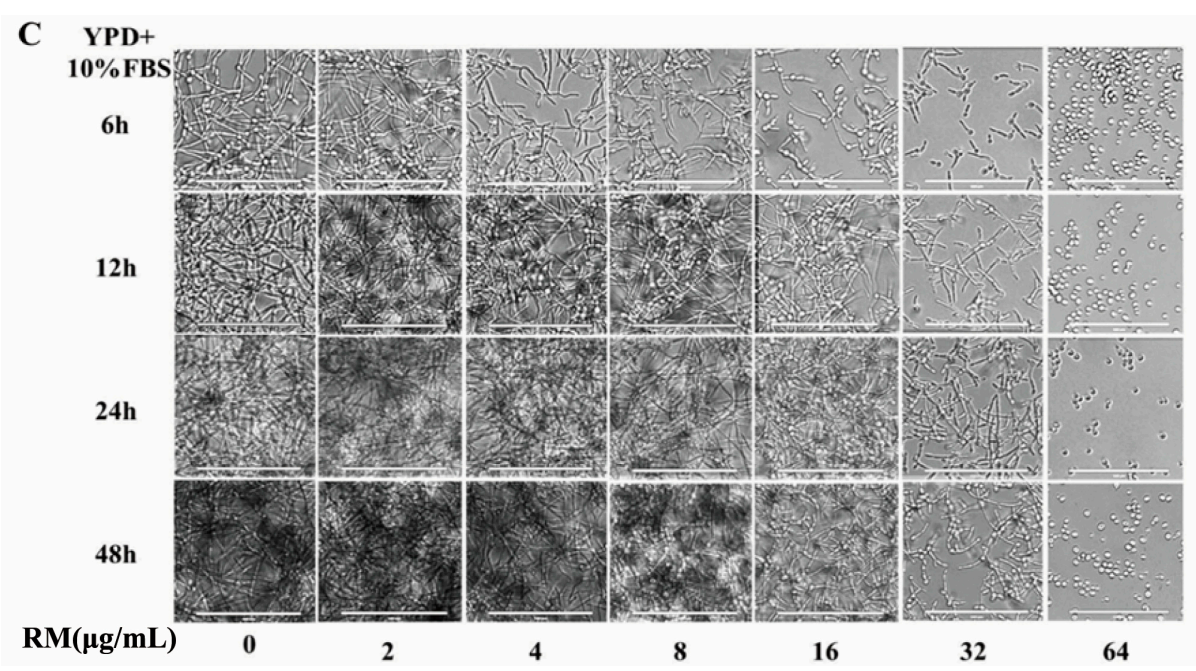

Figure 4. Effects of different concentrations of RM on hyphal formation. Exponentially growing C. albicans SC5314 cells were transferred to different hypha-inducing liquid media, (A) Spider medium; (B) Lee's medium; (C) YPD + 10\% FBS medium. The cellular morphology was photographed after incubation at $37{ }^{\circ} \mathrm{C}$ for $3.5 \mathrm{~h}$. Bar $=100 \mu \mathrm{m}$.

\subsection{RM Decreases CSH of C. albicans Biofilm}

In this study, the results showed that RM decreased CSH in a dose-dependent manner in four mediums (Spider, Lee's, RMPI1640 and YPD $+10 \%$ FBS) (Figure 5). The effect in Spider medium was the strongest and $4 \mu \mathrm{g} / \mathrm{mL}$ RM significantly decreased CSH from $0.75-0.17(p<0.001)$.

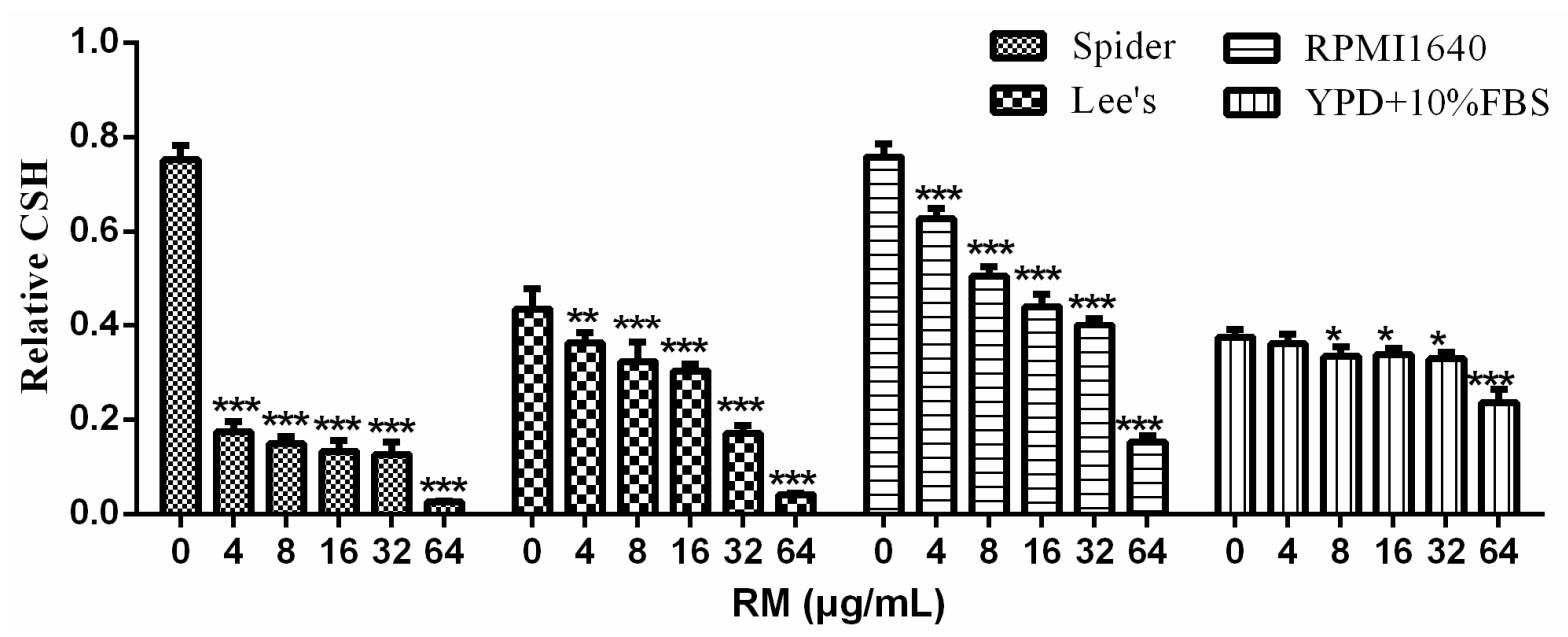

Figure 5. Effects of different concentrations of RM on CSH of C. albicans SC5314. The water-hydrocarbon two-phase assay was used to evaluate CSH. Standard deviations are based on three independent experiments. * $p<0.05 ; * * p<0.01 ; * * * p<0.001$.

\subsection{Exposure to RM Alters C. albicans Gene Transcription}

In order to understand the anti-biofilm mechanism of RM, we further investigated the expression changes of genes after RM treatment using real-time RT-PCR. The result showed that the biofilm-specific and hypha-specific genes such as YWP1, SAP5, SAP6, HWP1, ECE1 were up-regulated by 2.29, 5.99, 
2.27, 7.63, 15.44-fold, respectively, and EFG1 was down-regulated by 0.4013 -fold after $8 \mu \mathrm{g} / \mathrm{mL}$ RM treatment (Figure 6).

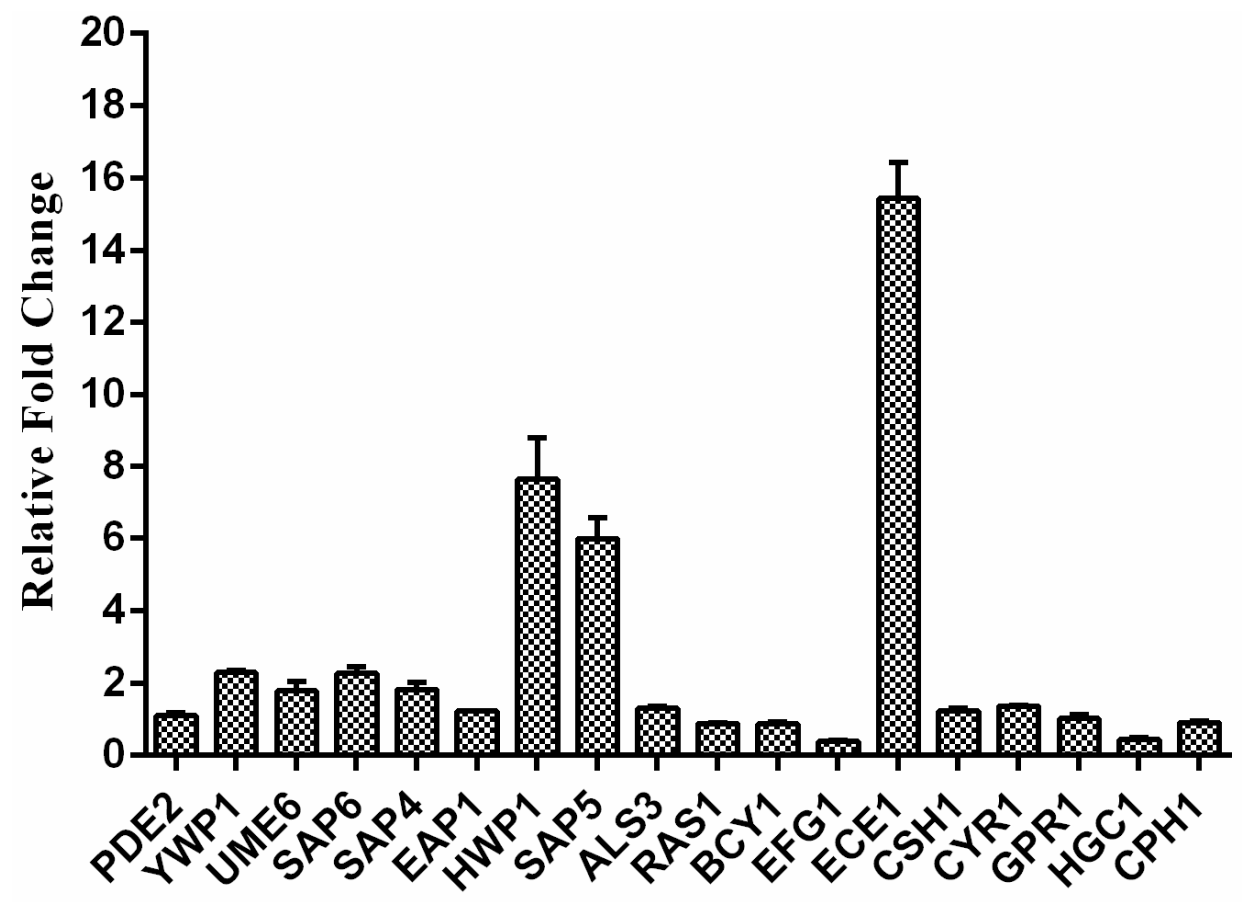

Figure 6. The expression changes of some important biofilm formation related genes after $8 \mu \mathrm{g} / \mathrm{mL}$ RM treatment. The C. albicans strain tested was SC5314. Gene expression was indicated as a fold change relative to that of the control group. 18S rRNA was used to normalize the expression data. Data are means \pm standard deviations from three experiments.

\subsection{Exogenous cAMP Reverts the Morphogenesis Defect Caused by RM}

Because some changed genes after RM treatment, such as HWP1, ECE1 and EFG1, were regulated by Ras/cAMP pathway and cAMP/PKA pathway [17], we performed exogenous cAMP supplement assay to verify that the antibiofilm mechanism of RM was related to the cAMP pathway. The result showed that exogenous cAMP could revert the morphogenesis defect of $C$. albicans cells caused by RM (Figure 7A,B). When $10 \mathrm{mM}$ cAMP were added in RM treated cultures, true hyphae were observed both in liquid and on solid Spider media (Figure 7A,B). This result indicates that RM may inhibit the biofilm formation by the cAMP pathway.

\subsection{Cytotoxicity Studies}

In this study, the results showed that RM had good cytotoxic effect against cancer cell lines SGC-7901, HT-29 and MGC-803 with IC50 of $0.844 \mathrm{mg} / \mathrm{L}(3.02 \mu \mathrm{M}), 1.279 \mathrm{mg} / \mathrm{L}(4.58 \mu \mathrm{M})$ and $0.631 \mathrm{mg} / \mathrm{L}$ $(2.26 \mu \mathrm{M})$, respectively. Besides, $\mathrm{RM}$ had no significant cytotoxicity against human umbilical vein endothelial cell with the $\mathrm{IC}_{50}$ of $43.047 \mathrm{mg} / \mathrm{L}(154.11 \mu \mathrm{M})$. This experiment confirmed that $\mathrm{RM}$ had lower cytotoxic effect for non-cancer cell lines. 


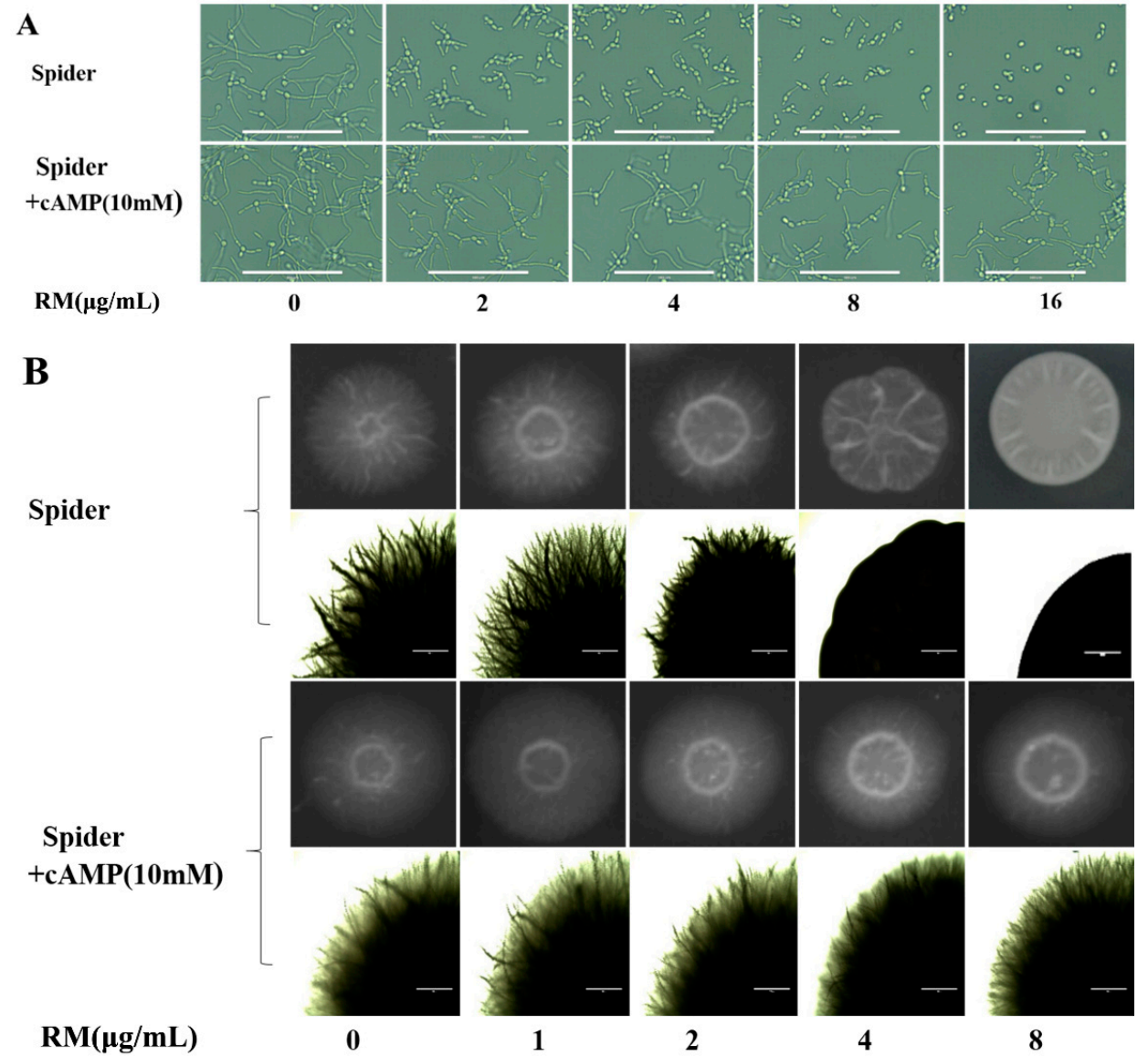

Figure 7. Addition of exogenous cAMP reverts the morphological transition defect of C. albicans SC5314 caused by RM. (A) Cells were incubated in liquid Spider medium supplemented with or without $10 \mathrm{mM}$ cAMP. The cells were incubated at $37{ }^{\circ} \mathrm{C}$ for $4 \mathrm{~h}$. Magnification 40×; (B) Hyphal formation on solid Spider medium plate with $10 \mathrm{mM}$ cAMP and different concentrations of RM, the cells were incubated at $37^{\circ} \mathrm{C}$ for 5 days.

\subsection{The Toxicity of RM on C. elegans Worms}

We investigated the toxicity of RM using health adult C. elegans worms. The results showed that at a range of concentrations, from 4-32 $\mu \mathrm{g} / \mathrm{mL}, \mathrm{RM}$ did not display toxicity, and all the worms looked healthy (Figure 8). At $64 \mu \mathrm{g} / \mathrm{mL} \mathrm{RM}$, only two worms died, and so high concentration of RM exhibits low toxicity. Nevertheless, RM has certain toxicity for the worms, the LD 50 of RM on C. elegans worms is $4096 \mu \mathrm{g} / \mathrm{mL}$. One gram per milliliter of RM can make all the worms die.

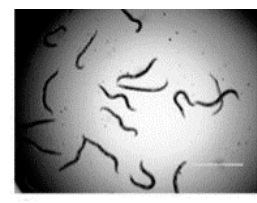

$\mathbf{R M}(\mu \mathrm{g} / \mathbf{m L})$

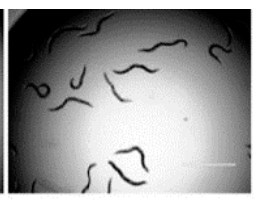

4

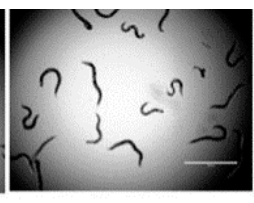

8

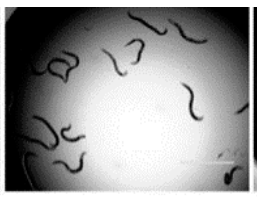

16

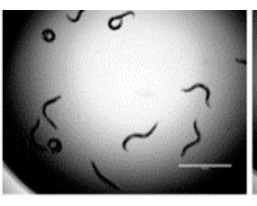

32

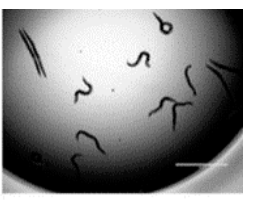

64

Figure 8. RM shows no significant toxicity on C. elegans glp-4; sek-1 nematodes. C. elegans glp-4; sek-1 nematodes were pipetted into 12-well plates that contained different concentrations of RM, incubated at $25^{\circ} \mathrm{C}$ for 6 days, and observed daily. 


\section{Discussion}

C. albicans is an opportunistic deep-infective fungi, still threatening our lives [18]. Most of established antifungal agents has been proved less than effective against $C$. albicans [19]. Therefore, the research and development of new antifungal agents is urgent. RM is a good therapeutic candidate that has been confirmed to have certain antifungal activity (MIC $80: 160 \mathrm{mg} / \mathrm{L}$ ) [13]. However, the effect of RM against C. albicans yeast cells and biofilm has not been elucidated. Consequently, in the current study, we focus on investigating the antifungal activity of RM against $C$. albicans biofilm and exploring the underlying mechanisms.

In this study, we found that RM had strong antimicrobial activity against common pathogens, such as A. fumigatus, S. aureus, C. glabratas, C. krusei, C. parapsilosis, and C. tropicalis. Unfortunately, RM showed weak activity against C. albicans (MIC80: 128-256 $\mu \mathrm{g} / \mathrm{mL}$ ). C. albicans forms biofilms easily, which results in C. albicans resistance to traditional antifungal agents [20]. Biofilm formation includes three stages: adhesion to biomaterial surfaces, growth to form an anchoring layer, and morphological transition to form a complex three-dimensional structure. To validate that RM has an anti-biofilm effect, we evaluated XTT reduction assay. Of note, we found that RM had strong effect against $C$. albicans biofilm. RM could inhibit the growth of biofilms in different liquid mediums, the strong-to-weak sequence of the effect of RM against C. albicans biofilms was Spider medium, Lee's medium and YPD + 10\% FBS medium. Furthermore, CLSM assay and SEM assay were established to observe biofilm formation directly, and the results were highly consistent with that obtained by XTT reduction assay. In summary, $\mathrm{RM}$ has a strong antibiofilm effect against $C$. albicans in vitro.

It was demonstrated that biofilm formation and the yeast-to-hypha morphological transition contributed to the pathogenic potential of $C$. albicans [21]. Our data showed that RM significantly inhibited the yeast-to-hypha morphological transition in both liquid and solid medium. The results indicate that the anti-biofilm activity effect of RM may be involved the growth of $C$. albicans hypha rather than yeast cells. In this study, our results showed that $4 \mu \mathrm{g} / \mathrm{mL}$ RM decreased CSH significantly $(p<0.001)$. CSH is one of the major pathogenic attributes of $C$. albicans and showed a positive correlation with adhesion [22]. It is the indicator for adhesion ability. These results indicate that the anti-biofilm effect of RM seems attributable to its anti-adhesion and anti-morphological transition activities.

In order to understand the anti-biofilm mechanism of RM, real-time RT-PCR assay was further performed. The data showed that the biofilm-specific and hypha-specific genes such as $Y W P 1, S A P 5$, $S A P 6, H W P 1, E C E 1$ were up-regulated and $E F G 1$ was down-regulated after $8 \mu \mathrm{g} / \mathrm{mL}$ RM treatment. $E C E 1$ is a hypha-specific gene and it encodes a membrane protein dependent on the cAMP pathway [23]. $H W P 1$ is a specific hyphal gene encoding a cell wall mannose protein, which is essential for normal growth of the mycelium. It also plays an important role in the pathogenesis of Candida infections. Mutation of the HWP1 gene results in incomplete biofilm formation [24]. ECE1 and HWP1 are regulated by Efg1. The protein is a transcription factor of the Ras/cAMP pathway and the cAMP/PKA pathway in hyphal development [17]. SAP5 and SAP6 encode secreted aspartic proteinase, which is expressed during hyphal growth. Expression of SAP5 and SAP6 also depends on Efg1 [25]. Based on this result, we speculated that the antibiofilm effect of RM might be related to the cAMP pathway. In this study, we fortunately found that exogenous cAMP could revert the hyphal morphogenesis defect of C. albicans cells caused by RM, the result further validated our speculation. However, the molecular mechanisms need to be further researched. 
Fungal and mammalian cells belong to eukaryotic cells, thus, it is very difficult to research and develop a new antifungal drug without toxicity in mammals. In this study, we used healthy C. elegans worms to study the toxicity of RM, and the results indicated that at the final concentration as high as $64 \mu \mathrm{g} / \mathrm{mL}$ RM exhibited low toxicity. Cytotoxic activities of RM have been studied in lung adenocarcinoma cancer cells (A549), cervical adenocarcinoma cancer cells (HeLa), breast cancer cells (MCF-7), prostate cancer cells (LNCaP and PC3) and two human non-cancer cell lines: human embryonic kidney 293(HEK293) and neonatal foreskin fibroblast (NFF) cells [26]. Interestingly, RM had selective cytotoxicity against cancer cells, such as: A549 cells (IC50 of $3.4 \mu \mathrm{M}$ ) and HeLa cells (IC50 of $4.0 \mu \mathrm{M}$ ). However, RM had less cytotoxicity against non-cancer cell lines, HEK293 cells (IC50 of $15.9 \mu \mathrm{M}$ ) and NFF cells (IC50 of 15.2 $\mu \mathrm{M}), \mathrm{RM}$ was selective against cancer cells because it had less inhibitory effect on the proliferation of non-cancer cells. It was noticable that the cells used in this study were not reported. The results revealed no significant cytotoxicity against human umbilical vein endothelial cell with the $\mathrm{IC}_{50}$ of $43.047 \mathrm{mg} / \mathrm{L}$ $(154.11 \mu \mathrm{M})$. This experiment further confirmed that RM had lower cytotoxic effect for non-cancer cell lines although additional studies in vivo need to be carried out to determine the toxicity of RM in mammals.

\section{Experimental Section}

\subsection{Strains and Growth Condition}

In this study, the international general laboratory strain: C. albicans SC5314 [27], Candida parapsilosis 90018, Staphylococcus aureus ATCC25913 [28], three clinical Candida spp. isolates: Candida glabrata 8535, Candida krusei 4996, Candida tropicalis 8915, one clinical Aspergillus fumigatus isolates: Aspergillus fumigatus 7544, (Changhai Hospital, Shanghai, China) and eight clinical MRSA isolates (Kunming General Hospital of Chengdu Military Region, Kunming, China) were used. All strains were identified according to standard morphological criteria. Prior to test, strains grew on sabouraud dextrose agar (SDA) at $30^{\circ} \mathrm{C}$ for $24 \mathrm{~h}$ and later subcultured in YPD ( $1 \%$ yeast extract, $2 \%$ peptone and $2 \%$ dextrose) liquid medium at $30{ }^{\circ} \mathrm{C}$ in a shaking incubator for $16 \mathrm{~h}$. RM (purity $\geq 96.8 \%$ ) was provided by Yunnan College of Traditional Chinese Medicine. It was dissolved in dimethyl sulfoxide (DMSO) for in vitro study.

\subsection{Antifungal Susceptibility Testing}

The minimal inhibitory concentration (MIC) was determined by the Clinical and Laboratory Standards Institute methods (M27-A3 and M27-S4) [29,30]. Briefly, the initial concentration in RPMI 1640 medium was $10^{3} \mathrm{CFU} / \mathrm{mL}$ for fungal suspension, and the final concentrations ranged from $4-256 \mu \mathrm{g} / \mathrm{mL}$ for RM. The 96-well microtiter plates were incubated at $35^{\circ} \mathrm{C}$ for $24 \mathrm{~h}$. The optical density at $630 \mathrm{~nm}\left(\mathrm{OD}_{630}\right)$ was measured to determine the growth inhibition. Each strain was tested in triplicate. The MIC 50 was defined as the lowest concentration of the drugs that inhibited growth by $50 \%$ compared with the drug-free growth control.

\subsection{Time-Growth Curves Assay}

Exponentially growing C. albicans SC5314 were suspended in YPD medium to the starting OD 600 of 0.2 (about $2.5 \times 10^{6}$ cells $/ \mathrm{mL}$ ). Then the suspensions were added in different concentrations of RM and 
cultured at $30{ }^{\circ} \mathrm{C}, 200 \mathrm{rpm}$. Cells were counted at the predetermined time points $(0,2,4,6,8,12,24 \mathrm{~h})$. Three independent experiments were performed [31].

\subsection{In Vitro Biofilm Formation Assay}

The in vitro biofilm formation assay [32] was performed in a 96-well tissue culture plate by seeding $100 \mu \mathrm{L} 1.0 \times 10^{6} \mathrm{CFU} / \mathrm{mL}$ C. albicans cells suspension in Spider medium[33], Lee's medium, YPD $+10 \%$ FBS (fetal bovine serum) [34] and RPMI 1640 medium and incubated at $37{ }^{\circ} \mathrm{C}$. After 90-min adhesion, the medium was removed and the fresh medium with different concentrations of RM was added. And the plates were incubated statically at $37^{\circ} \mathrm{C}$ for $24 \mathrm{~h}$. Then the biofilms were washed with phosphate-buffered saline (PBS) and added $200 \mu \mathrm{L}$ 2, 3-bis-(2-methoxy-4-nitro-5-sulfophenyl)2H-tetrazolium-5-carboxanilide (XTT)/menadione. After incubating at $37{ }^{\circ} \mathrm{C}$ for $2 \mathrm{~h}$ in a dark room, the formed biofilms were determined using XTT reduction assay.

\subsection{Confocal Laser Scanning Microscopy (CLSM) Assay}

CLSM was performed to determine the inhibitory effect of RM on biofilm formation. The confocal laser scanning special culture dishes were inoculated with C. albicans 5314 at $37{ }^{\circ} \mathrm{C}$ for 90 min to allow adhesion. Then biofilms were stained with $100 \mu \mathrm{g} / \mathrm{mL}$ of calcofluor white (Fluka, 18909) in the dark. After that, samples were washed with $2 \mathrm{~mL}$ PBS and observed under a Leica TCS SP2 confocal laser scanning microscopy (excitation wavelength $355 \mathrm{~nm}$; emission wavelength $433 \mathrm{~nm}$ ) [35].

\subsection{Scanning Electron Microscopy (SEM) Assay}

For SEM assay, the biofilms were formed on silicone pads in Spider medium at $37^{\circ} \mathrm{C}$ for 90 min [36]. After removing non-adherent cells, and the fresh medium with different concentrations of RM was added. Then, the samples were incubated at $37{ }^{\circ} \mathrm{C}$ for $24 \mathrm{~h}$. Subsequently, biofilms were placed in fixed liquid specific for electron microscopy (G1020, Goodbio Technology CO., Ltd, Wuhan, China) for $2 \mathrm{~h}$ and dehydrated in an ascending ethanol series and dried naturally. The dry specimens were coated with gold and observed through an EVO MA1O SEM (Carl Zeiss GmbH, Eppelheim, Germany).

\subsection{Yeast-to-Hyphae Transition Assays}

Exponentially growing C. albicans SC5314 were incubated with different concentrations of RM in hypha-inducing liquid medium at $37^{\circ} \mathrm{C}$ for $6,12,24,48 \mathrm{~h}$ respectively and on solid medium(Spider, Lee's, YPD $+10 \% \mathrm{FBS}$ ) at $37^{\circ} \mathrm{C}$ for 5 days. The cells were observed and photographed by inverted phase contrast microscope $\left(\mathrm{AMG}^{\circledR}\right.$ EVOS xl, Life Technologies, Carlsbad, CA, USA).

\subsection{Cellular Surface Hydrophobicity (CSH) Assay}

CSH assay was based on water-hydrocarbon interface assay as described by Stephen A et al. [37]. The biofilms were harvested and suspended in YPD medium at $\mathrm{OD}_{600}$ of 1.0. A total of $1.2 \mathrm{~mL}$ of this suspension plus $0.3 \mathrm{~mL}$ octane was placed in a sterile glass tube and vortexed for $3 \mathrm{~min}$. OD 600 of the aqueous phase was recorded after settled for $10 \mathrm{~min}$. $\mathrm{OD}_{600}$ of the suspension without the octane overlay 
was determined as control. Relative hydrophobicity (\%) was obtained as [(OD600 of the control minus OD 600 of the treatment)/OD 600 of the control] $\times 100$.

\subsection{Real-Time RT-PCR}

Real-time RT-PCR was performed according to the method described previously [38,39]. The biofilms of C. albicans 5314 were harvested and used for the total RNA extraction. Triplicate independent experiments were performed for each sample. The total RNA was extracted using Fungal RNAout kit (TIANDS, Beijing, China). 1st-strand cDNA was synthesized by reverse transcription reaction using a reverse transcription kit (TaKaRa, Biotechnology, Dalian, China). SYBR Green I (TaKaRa) and LightCycler Real-Time PCR system (Roche diagnostics GmbH, Mannheim, Germany) were used for Real-time PCR. Forward primer and reverse primer were listed in Supplementary Table S1. 18S rRNA played the role of internal control. The thermal cycling comprised four steps, an initial step at $95{ }^{\circ} \mathrm{C}$ for $2 \mathrm{~min}, 40$ cycles at $95^{\circ} \mathrm{C}$ for $10 \mathrm{~s}, 60^{\circ} \mathrm{C}$ for $20 \mathrm{~s}$, and $72{ }^{\circ} \mathrm{C}$ for $30 \mathrm{~s}$. Finally, the gene expression level was calculated using the formula $2^{-\Delta \Delta \mathrm{Cr}}$.

\subsection{Exogenous Cyclic AMP (cAMP) Supplement Assay}

Exponentially growing $C$. albicans SC5314 cells were resuspended in Spider liquid medium at $5.0 \times 10^{5} \mathrm{CFU} / \mathrm{mL}$. Then, different concentrations of RM with or without cAMP $(10 \mathrm{mM})$ were added. After that, the samples were incubated at $37^{\circ} \mathrm{C}$ for $4 \mathrm{~h}$. Simultaneously, $5.0 \times 10^{2} \mathrm{CFU} / \mathrm{mL}$ C. albicans SC5314 cells were colonized on Spider solid medium at $37^{\circ} \mathrm{C}$ for 5 days. The cells were observed and photographed by inverted phase contrast microscope $\left(\mathrm{AMG}^{\circledR} \mathrm{EVOS} \mathrm{xl}\right)$.

\subsection{Cytotoxicity Studies}

Three cancer cells (human gastric cancer cell line SGC-7901, human colon cancer cell line HT-29, human gastric cancer cell line MGC-803) and one normal cell (human umbilical vein endothelial cell) were employed in cytotoxicity tests. The cytotoxicity of RM was evaluated as the concentration of $50 \%$ cellular cytotoxicity $\left(\mathrm{IC}_{50}\right)$ [40].

\subsection{Toxicity Evaluation using C. elegans Worms}

C. elegans glp-4; sek-1 adult nematodes were used to evaluate the toxicity of RM. Briefly, the nematodes were moved from Escherichia coli $\mathrm{OP}_{50}$ to pathogen-free liquid medium that contained different concentrations of RM or $1 \%$ DMSO. Then, the worms were incubated at $25{ }^{\circ} \mathrm{C}$ for 6 days and observed daily.

\section{Conclusions}

In the present study, we found that RM obviously inhibits yeast-to-hypha transition and the C. albicans biofilm formation in vitro, but it has no fungicidal effect on planktonic $C$. albicans cells, and the anti-biofilm mechanism may be related to the cAMP pathway. In addition, RM is a safe alkaloid for human non-cancer cell lines. Whether the anti-biofilm effect of RM is applicable in clinical management requires further study, and in vivo activities of RM against $C$. albicans need to be investigated. 


\section{Supplementary Materials}

Supplementary materials can be accessed at: http:/www.mdpi.com/1420-3049/20/10/17913/s1.

\section{Acknowledgments}

This work was supported by the National Natural Science Foundation of China (grant number 81173643 , 81330083, 81470158, 81460560), the China National 973 Program (grant number 2013CB531602) and the Science Foundation of Yunnan Province (grant number 2010CD114).

\section{Author Contributions}

C.M., F.D., L.Y., Y.J. and G.X. performed research; C.M., F.D., G.H., J.H., C.W., G.R. and G.X. analyzed the data and C.M. and G.X. wrote the paper. All the authors read and approved the final manuscript.

\section{Conflicts of Interest}

The authors declare no conflict of interest.

\section{References}

1. Gow, N.A.; van de Veerdonk, F.L.; Brown, A.J.; Netea, M.G. Candida albicans morphogenesis and host defence: Discriminating invasion from colonization. Nat. Rev. Microbiol. 2011, 10, 112-122.

2. Li, D.D.; Xu, Y.; Zhang, D.Z.; Quan, H.; Mylonakis, E.; Hu, D.D.; Li, M.B.; Zhao, L.X.; Zhu, L.H.; Wang, Y.; et al. Fluconazole assists berberine to kill fluconazole-resistant Candida albicans. Antimicrob. Agents Chemother. 2013, 57, 6016-6027.

3. Sudbery, P.E. Growth of Candida albicans hyphae. Nat. Rev. Microbiol. 2011, 9, 737-748.

4. Zhao, L.X.; Li, D.D.; Hu, D.D.; Hu, G.H.; Yan, L.; Wang, Y.; Jiang, Y.Y. Effect of tetrandrine against Candida albicans biofilms. PLoS ONE 2013, 8, e79671.

5. Ramage, G; Saville, S.P.; Thomas, D.P.; Lopez-Ribot, J.L. Candida biofilms: An update. Eukaryot. Cell 2005, 4, 633-638.

6. Chandra, J.; Mukherjee, P.K.; Leidich, S.D.; Faddoul, F.F.; Hoyer, L.L.; Douglas, L.J.; Ghannoum, M.A. Antifungal resistance of candidal biofilms formed on denture acrylic in vitro. J. Dent. Res. 2001, 80, 903-908.

7. Valiente, M.; D’Ocon, P.; Noguera, MA.; Cassels, B.K.; Lugnier, C.; Ivorra, M.D. Vascular activity of $(-)$-anonaine, $(-)$-roemerine and (-)-pukateine, three natural $6 \mathrm{a}(R)$-1, 2-methylenedioxyaporphines with different affinities for $\alpha 1$-adrenoceptor subtypes. Planta Med. 2004, 70, 603-609.

8. Lin, R.J.; Wu, M.H.; Ma, Y.H.; Chung, L.Y.; Chen, C.Y.; Yen, C.M. Anthelmintic activities of aporphine from Nelumbo nucifera Gaertn. cv. Rosa-plena against Hymenolepis nana. Int. J. Mol. Sci. 2014, 15, 3624-3639.

9. Baghdikian, B.; Mahiou-Leddet, V.; Bory, S.; Bun, S.S.; Dumetre, A.; Mabrouki, F.; Hutter, S.; Azas, N.; Ollivier, E. New antiplasmodial alkaloids from Stephania rotunda. J. Ethnopharmacol. 2013, 145, 381-385. 
10. You, M.; Wickramara, D.B.; Silva, G.L.; Chai, H.; Chagwedera, T.E.; Farnsworth, N.R.; Cordell, G.A.; Kinghorn, A.D.; Pezzuto, J.M. (-)-Roemerine, an aporphine alkaloid from Annona senegalensis that reverses the multidrug-resistance phenotype with cultured cells. J. Nat. Prod. 1995, 58, 598-604.

11. Munusamy, V.; Yap, B.K.; Buckle, M.J.; Doughty, S.W.; Chung, L.Y. Structure-based identification of aporphines with selective 5-HT (2A) receptor-binding activity. Chem. Biol. Drug Des. 2013, 81, 250-256.

12. Tsai, I.L.; Liou, Y.F.; Lu, S.T. Screening of isoquinoline alkaloids and their derivatives for antibacterial and antifungal activities. Kaohsiung J. Med. Sci. 1989, 5, 132-145.

13. Rao, G.X.; Zhang, S.; Wang, H.M.; Li, Z.M.; Gao, S.; Xu, G.L. Antifungal alkaloids from the fresh rattan stem of Fibraurea recisa Pierre. J. Ethnopharmacol. 2009, 123, 1-5.

14. Liu, Y.Q.; He, G.H.; Li, H.L.; He, J.C.; Feng, E.F.; Bai, L.; Wang, C.Y.; Xu, G.L. Plasma pharmacokinetics and tissue distribution study of roemerine in rats by liquid chromatography with tandem mass spectrometry (LC-MS/MS). J. Chromatogr. B Anal. Technol. Biomed. Life Sci. 2014, 969, 249-255.

15. Cullen, P.J.; Sprague, G.J. The regulation of filamentous growth in yeast. Genetics 2012, 190, $23-49$.

16. Lu, Y.; Su, C.; Liu, H. Candida albicans hyphal initiation and elongation. Trends Microbiol. 2014, 22, 707-714.

17. Bockmuhl, D.P.; Ernst, J.F. A potential phosphorylation site for an A-kinase in the Efg1 regulator protein contributes to hyphal morphogenesis of Candida albicans. Genetics 2001, 157, 1523-1530.

18. Teodoro, J.G.; Heilman, D.W.; Parker, A.E.; Green, M.R. The viral protein Apoptin associates with the anaphase-promoting complex to induce G2/M arrest and apoptosis in the absence of p53. Genes Dev. 2004, 18, 1952-1957.

19. Liu, S.; Hou, Y.; Chen, X.; Gao, Y.; Li, H.; Sun, S. Combination of fluconazole with non-antifungal agents: A promising approach to cope with resistant Candida albicans infections and insight into new antifungal agent discovery. Int. J. Antimicrob. Agents 2014, 43, 395-402.

20. Chandra, J.; Kuhn, D.M.; Mukherjee, P.K.; Hoyer, L.L.; McCormick, T.; Ghannoum, M.A. Biofilm formation by the fungal pathogen Candida albicans: Development, architecture, and drug resistance. J. Bacteriol. 2001, 183, 5385-5394.

21. Mayer, F.L.; Wilson, D.; Hube, B. Candida albicans pathogenicity mechanisms. Virulence 2014, 4, 119-128.

22. Samaranayake, Y.H.; Wu, P.C.; Samaranayake, L.P.; So, M. Relationship between the cell surface hydrophobicity and adherence of Candida krusei and Candida albicans to epithelial and denture acrylic surfaces. APMIS 1995, 103, 707-713.

23. Fan, Y.; He, H.; Dong, Y.; Pan, H. Hyphae-specific genes HGC1, ALS3, HWP1, and ECE1 and relevant signaling pathways in Candida albicans. Mycopathologia 2013, 176, 329-335.

24. Nobile, C.J.; Schneider, H.A.; Nett, J.E.; Sheppard, D.C.; Filler, S.G.; Andes, D.R.; Mitchell, A.P. Complementary adhesin function in C. albicans biofilm formation. Curr. Biol. 2008, 18, 1017-1024.

25. Moazeni, M.; Khoramizadeh, M.R.; Teimoori-Toolabi, L.; Noorbakhsh, F.; Rezaie, S. The effect of EFG1 gene silencing on down-regulation of SAP5 gene, by use of RNAi technology. Acta Med. Iran. 2014, 52, 9-14.

26. Tran, T.D.; Pham, N.B.; Fechner, G.; Quinn, R.J. Chemical investigation of drug-like compounds from the Australian tree, Neolitsea dealbata. Bioorg. Med. Chem. Lett. 2010, 20, 5859-5863. 
27. Krause, J.; Geginat, G.; Tammer, I. Prostaglandin E2 from Candida albicans stimulates the growth of Staphylococcus aureus in mixed biofilms. PLoS ONE 2015, 10, e 0135404.

28. Clinical and Laboratory Standards Institute (CLSI). M07-A9: Methods for Dilution Antimicrobial Susceptibility Tests for Bacteria That Grow Aerobically; Approved Standard-Ninth Edition; CLSI Publication: Wayne, PA, USA, 2012.

29. Clinical and Laboratory Standards Institute (CLSI). M27-A3: Reference Method for Broth Dilution Antifungal Susceptibility Testing of Yeasts; Approved Standard-Third Edition; CLSI Publication: Wayne, PA, USA, 2009.

30. Clinical and Laboratory Standards Institute (CLSI). M27-S4: Reference method for Broth Dilution Antifungal Susceptibility Testing of Yeasts; Fourth Informational Supplement; CLSI Publication: Wayne, PA, USA, 2012.

31. Dhamgaye, S.; Devaux, F.; Vandeputte, P.; Khandelwal, N.K.; Sanglard, D.; Mukhopadhyay, G.; Prasad, R. Molecular mechanisms of action of herbal antifungal alkaloid berberine, in Candida albicans. PLoS ONE 2014, 9, e104554.

32. Li, D.D.; Zhao, L.X.; Mylonakis, E.; Hu, G.H.; Zou, Y.; Huang, T.K.; Yan, L.; Wang, Y.; Jiang, Y.Y. In vitro and in vivo activities of pterostilbene against Candida albicans biofilms. Antimicrob. Agents Chemother. 2014, 58, 2344-2355.

33. Daniels, K.J.; Park, Y.N.; Srikantha, T.; Pujol, C.; Soll, D.R. Impact of environmental conditions on the form and function of Candida albicans biofilms. Eukaryotic Cell 2013, 12, 1389-1402.

34. Maidan, M.M.; De Rop, L.; Serneels, J.; Exler, S.; Rupp, S.; Tournu, H.; Thevelein, J.M.; van Dijck, P. The G protein-coupled receptor Gpr1 and the Galpha protein Gpa2 act through the cAMP-protein kinase A pathway to induce morphogenesis in Candida albicans. Mol. Biol. Cell 2005, 16, 1971-1986.

35. Cao, Y.Y.; Dai, B.; Wang, Y.; Huang, S.; Xu, Y.; Cao, Y.; Gao, P.; Zhu, Z.; Jiang, Y. In vitro activity of baicalein against Candida albicans biofilms. Int. J. Antimicrob. Agents 2008, 32, 73-77.

36. Nobile, C.J.; Andes, D.R.; Nett, J.E.; Smith, F.J.; Yue, F.; Phan, Q.T.; Edwards, J.E.; Filler, S.G.; Mitchell, A.P. Critical role of Bcr1-dependent adhesins in C. albicans biofilm formation in vitro and in vivo. PLoS Pathog. 2006, 2, e63.

37. Klotz, S.A.; Drutz, D.J.; Zajic, J.E. Factors governing adherence of Candida species to plastic surfaces. Infect. Immun. 1985, 50, 97-101.

38. Sun, L.M.; Cheng, A.X.; Wu, X.Z.; Zhang, H.J.; Lou, H.X. Synergistic mechanisms of retigeric acid B and azoles against Candida albicans. J. Appl. Microbiol. 2010, 108, 341-348.

39. Li, D.D.; Wang, Y.; Dai, B.D.; Li, X.X.; Zhao, L.X.; Cao, Y.B.; Yan, L.; Jiang, Y.Y. ECM17-dependent methionine/cysteine biosynthesis contributes to biofilm formation in Candida albicans. Fungal Genet. Biol. 2013, 51, 50-59.

40. Kim, H.; Jang, J.H.; Kim, S.C.; Cho, J.H. De novo generation of short antimicrobial peptides with enhanced stability and cell specificity. J. Antimicrob. Chemother. 2014, 69, 121-132.

Sample Availability: Samples of the isolated compounds are available from the authors.

(C) 2015 by the authors; licensee MDPI, Basel, Switzerland. This article is an open access article distributed under the terms and conditions of the Creative Commons Attribution license (http://creativecommons.org/licenses/by/4.0/). 